REOP. Vol. 13, No 1, $1^{\text {er }}$ Semestre, 2002, pp. 121-133

\title{
LA ORIENTACIÓN LABORAL A DESEMPLEADOS INSCRITOS EN LAS OFICINAS DE EMPLEO DEL INEM
}

\author{
THE JOB ORIENTACION SERVICE TO THE UNEMPLOYED \\ PEOPLE OF INEM
}

\author{
Josefina Hernández Fernández* y Juana Ruiz del Cerro** \\ Universidad de Murcia
}

\section{RESUMEN}

En este estudio se ofrece una descripción de los desempleados que pueden acudir a un Servicio de Orientación Laboral.

Aunque existen estudios que abordan el fenómeno del desempleo, del mismo modo que hay multitud de programas de Orientación Laboral, no hemos encontrado trabajos de investigación que muestren la realidad de los demandantes inscritos en las Oficina de Empleo. En este estudio se describen los perfiles más característicos, sus condicionantes, necesidades o intereses profesionales.

Atendiendo a estos factores en el Servicio de Orientación de una Oficina de Empleo de Murcia se ha realizado una clasificación, en ella aparecen tres grupos bien diferenciados: universitarios, no universitarios y perceptores de rentas para la inserción laboral.

La propuesta de esta investigación es que, aprovechando la realidad descrita, se elaboren estrategias de apoyo -relacionadas con la orientación para el empleo- con actividades acopladas a las características de los colectivos señalados, atendiendo, también, a especificaciones como la edad, el género, la formación o la experiencia profesional.

Palabras clave: desempleo, paro, orientación-laboral, programas-de-orientación.

\section{ABSTRACT}

The following study describes a job orientation service to a kind of unemployed people.

* Orientadora Laboral en una Oficina de Empleo del INEM en Murcia. Su tesis doctoral: La Orientación como medida correctora del desempleo de larga duración, y sus publicaciones, se centran en el Desempleado y los Programas de Orientación. Actualmente profesora Asociada en Dto. de Psicología Social de Universidad de Murcia. (investigadora principal).

** Profesora titular de Orientación Educativa. Ha publicado varios libros sobre Orientación Educativa, y sus líneas de investigación se centran en la Orientación Profesional y la Evaluación de Programas de Orientación. 
Although there are studies to the unemployed and job orientation service there is no counselling programmes that shows the truth of the unemployed registered in the job centre. So, an analysis on the features of those groups found in an agency of Murcia is described.

Combining such data as age, gender, instruction, work experience there are the characteristics of three groups demanding employment: graduate students, undergraduates and salary recipients for reemployment have been defined. These data can help to create new programs.

Key Words: unemployment, job-orientation, counseling-programmes

\section{Introducción}

Sabemos que el desempleo es un fenómeno que tiene un interés preferente en foros y debates de política social (a nivel internacional, nacional, regional o local). Con ello se está generando multitud de ayudas para el desarrollo de programas dirigidos a orientar en materia laboral a los distintos colectivos, pero no existen descripciones de la situación de cada uno de ellos: sus intereses, condicionantes, necesidades o debilidades.

Por este motivo nos interesaba, además de repasar los marcos teóricos, plasmar nuestra experiencia y publicarla, para compartir con otros técnicos de orientación la información, apoyar su formación o requerir su apoyo y colaboración para elaborar los programas de apoyo a la inserción laboral para los distintos subgrupos que coexisten en el desempleo.

Éramos conscientes de que había realidades diferentes en los demandantes de empleo y nos lanzamos a describir las características encontradas, en concreto se analizaron los 108 demandantes que acudieron al Servicio de Orientación de la Oficina de Empleo MurciaJorge Juan durante el primer semestre del año 2000. Elegimos este lugar debido a que teníamos aquí nuestro puesto de trabajo como orientadores laborales.

Esta Oficina es una de las más concurridas de la Región de Murcia, ya que atiende a unos 5.000 demandantes de empleo -de Murcia capital y pedanías-, y en su base de datos se pueden encontrar demandantes de todos los sectores profesionales pero mayoritariamente del sector servicios.

\section{Metodología del trabajo de investigación}

Para recoger los datos, que sirven de fundamentación a este estudio, después de atender a cada usuario, individualmente, se recogieron sus características personales y profesionales - manteniendo el anonimato de los usuarios- en fichas individuales. Cada ficha, fue identificad con un número y contenía la edad, el género, la formación, la experiencia profesional, las expectativas laborales y el servicio de orientación ofrecido.

Como podrá comprobarse el número de sujetos que formaron cada grupo no es idéntico (se trabajó con 15 no universitarios, 28 universitarios y 65 perceptores de la Renta para la Inserción), ya que se incluyó a todos los demandantes de empleo que acudieron en ese periodo -enero a julio de 2000- al servicio de Orientación de esta Oficina. Unos lo hicieron de forma voluntaria y otros -los preceptores de ayudas económicas para la inserción- por requerimiento del INEM. 
Una vez registrados dichos datos, se procedió a analizar las diferencias fundamentales, contrastándolas con teorías o valoraciones de expertos que han abordado el fenómeno del desempleo en otros contextos, como Goodale (1982), Blanch (1990), Watt (1997), Villamarzo (1994) o Puchol (1994), y en los que encontramos un fiel reflejo de la realidad que estábamos estudiando en Murcia.

\section{Fundamentación teórica}

\section{El fenómeno del desempleo}

Creemos, como Villamarzo (1994:13-14), que para mantener un contacto idóneo con la realidad es necesario disfrutar de amor y trabajo, y que este último es el mejor profiláctico y antídoto de multitud de problemas psicopatológicos.

El trabajo es, por tanto, en esta sociedad contemporánea y occidental, un medio de subsistencia que, además, puede aportar identidad personal, orden al tiempo existencial, integra en la sociedad y, sobre todo en los empleos de cuello blanco, puede ofrecer una importante fuente de satisfacción personal.

Visto así, se entiende que el desempleo puede conducir a la desesperación. Aunque sabemos que los desempleados no es un grupo homogéneo y que no todos esperan con el mismo entusiasmo un puesto de trabajo, estamos de acuerdo con Blanch (1990: 144) cuando nos señala que:

«Resulta [...] razonable una explicación de la depresión en el desempleo como desenlace lógico del proceso [...] quien, habiendo discurrido normalmente por los cauces establecidos a lo largo de su proceso de socialización, [...] se halla aparcado en la vía muerta de la desocupación laboral».

En todos los países occidentales, conscientes de esta problemática, la del desempleo, se están tomando diversas medidas -incluidas en la línea de políticas «activas» contra el desempleo- que ofrecen reciclaje profesional y/u orientación laboral a los demandantes de empleo con el fin de conducirles hacia una mejora de su situación.

\section{Tipologías en el empleo y desempleo}

Es difícil realizar un análisis real de las características del desempleo en nuestro país, pues aunque los datos estadísticos, mes a mes, invaden periódicos y medios de comunicación, todos los recibimos con cierto escepticismo, ya que en las listas del INEM sabemos que no están todos los que son ni son todos los que están.

Además, dentro de los que están inscritos, habría que distinguirlos, según el tipo de jornada que solicitan y sus objetivos de cara al trabajo, ya que unos buscan trabajo a jornada completa pero otros se contentarían con un trabajo a tiempo parcial, e incluso algunos no desean encontrar ningún trabajo, sino que el objetivo de su inscripción como demandantes de empelo es la de cumplir los requisitos para acceder a los cursos de Formación Ocupacional, organizados de forma gratuita para todos ellos. 
También pueden establecerse algunas diferencias, si unimos características como edad y género aparecen distintas tipologías; por ejemplo la de las amas de casa que quieren incorporarse al mundo laboral después de la edad de la crianza, y que están a una distancia considerable -por debajo- respecto a los hombres de su misma edad y formación (Puchol, 1994:208).

Por otra parte, si analizamos el nivel de aspiraciones, que también suele ir relacionado con la edad, encontramos otro distintivo fundamental ya que tiene connotaciones distintas en:

a) Los desempleados menores de 30 años, que en los primeros empleos necesitan demostrar lo que saben o valen, aunque sea a cambio de un insignificante salario.

b) Los mayores de 50 años, que tratan de buscar, sobre todo, buenas condiciones para su jubilación: altas cotizaciones y un salario acorde a su categoría profesional.

c) Y por último, los que están en medio, en torno a los de 40 años, a los que no es posible seducirles con un empleo de baja retribución, a pesar de que saben que no es fácil encontrar trabajo a partir de su edad.

También hay un colectivo que Blanch (1990) define como no ocupables debido su edad, avanzada, y bajo nivel cultural o profesional.

Dentro de este colectivo hay, también un tipo de desempleados voluntarios, llamados también "parásitos del bienestar», que mantienen una actitud de indefensión, que no siempre están dispuestos a abandonar. Y cercanos a éstos, están otros que sí quieren trabajar, pero sólo para conseguir un dinero extra (sobre todo estudiantes y mujeres dedicadas a la familia).

Y por último, como señala Goodale (1982: 173) los auténticos buscadores de empleo, que permanentemente cambian su cualificación profesional para adaptarse a las necesidades del mercado laboral, implicándose en una carrera que no termina nunca, la carrera proteica.

\section{Los servicios de Orientación Laboral}

Parece indiscutible la diferencia entre un desempleado que quiere y sabe cómo buscar empleo y otro que espera pasivamente. Los servicios de Orientación Laboral pretenden colocar al demandante de empleo en las mejores condiciones para que pueda buscarlo activamente y consiga sus objetivos profesionales.

La Orientación Laboral, por otros llamada Asesoramiento (Watt, 1997), pretende ayudar a los desempleados a salir lo antes posible del desempleo, sirviéndose de diversos programas de apoyo.

Según la entidad, disponibilidad de presupuestos, medios, etc., se han desarrollado multitud de programas, que vamos a presentar clasificados en cuatro grandes bloques, los mismos que se han ofrecido a los demandantes en la Oficina de Empleo en la que se realizado este estudio:

a) Información: Consiste en ofrecer datos sobre el mercado laboral al demandante, para que éste conozca las ofertas del sector u ocupación en el que busca empleo, cursos de formación reglada u ocupacional que puedan mejorar su perfil profesional, direcciones de interés, servicios complementarios de su zona, etc. 
b) Instrumentos de Búsqueda: Con este programa, se ayuda a redactar currículos, descubrir el modo de afrontar entrevistas de selección o realizar tests psicotécnicos.

c) Análisis Vocacional: Se utiliza con demandantes que necesiten clarificar sus intereses profesionales, reflexionar sobre su escala de valores, establecer prioridades o planes para la acción de búsqueda.

d) Motivación de Logro: El objetivo de estas actividades es profundizar en el dominio de habilidades sociales, la asertividad, autoestima o expectativas de logro respecto a la consecución de un trabajo acorde a su perfil profesional y necesidades del mercado laboral.

La variedad de tipologías, problemáticas y circunstancias que envuelven a cada demandante de empleo exige que para cada uno de los usuarios atendidos se seleccione una modalidad de servicio, posiblemente combinando actividades de diversos programas.

\section{Descripción de la experiencia}

A continuación vamos a tratar de describir los tres colectivos (universitarios, no universitarios y preceptores de renta activa para la inserción) en los que hemos agrupado a los demandantes que han acudido, durante el primer semestre del año 2000, al servicio de Orientación de la Oficina de Empleo de Murcia.-Jorge Juan.

\section{Orientados no Universitarios}

El primer grupo lo hemos denominado no universitarios porque encontramos que tienen unas peculiaridades que lo separan del resto.

Nuestros datos los hemos recogido de las 15 personas que acudieron con las siguientes condiciones: sus edades están comprendidas entre los 22 y los 50 años y han acudido al servicio de forma voluntaria. Encontramos una gran laguna entre los 33 y los 38 años; y, puesto que este servicio ha sido solicitado mayoritariamente por mujeres, puede que esta falta de demandantes esté relacionada con la edad de la crianza (Gráfico 1).

Su formación es mayoritariamente de Bachillerato, aunque algunos tienen Certificado y Graduado de Escolaridad o Formación Profesional de Primero y Segundo grado, en la proporción señalada en el gráfico 2.

La experiencia profesional de este grupo está vinculada tanto a oficios cualificados como no cualificados, y son: limpiadora, asistenta de hogar, cajera, camarera, ordenanza, telefonista, modista, etc. Parece ser que las personas más jóvenes son las que han recorrido mayor variedad de puestos.

Estudiando su disponibilidad para trabajar de forma inmediata, vemos que hay ligeras diferencias intragrupo, ya que las más jóvenes (de 22 a 33 años), ponen menos requisitos y tienen menos dificultades que las mayores.

En el análisis de las narraciones, para entender sus circunstancias particulares, encontramos que algunas están dispuestas a aceptar un trabajo de forma inmediata (este grupo representa el $47 \%$ del total), y el motivo es que necesitan trabajar para vivir, para mantener la custodia de sus hijos o porque consideran que es una necesidad vital. 


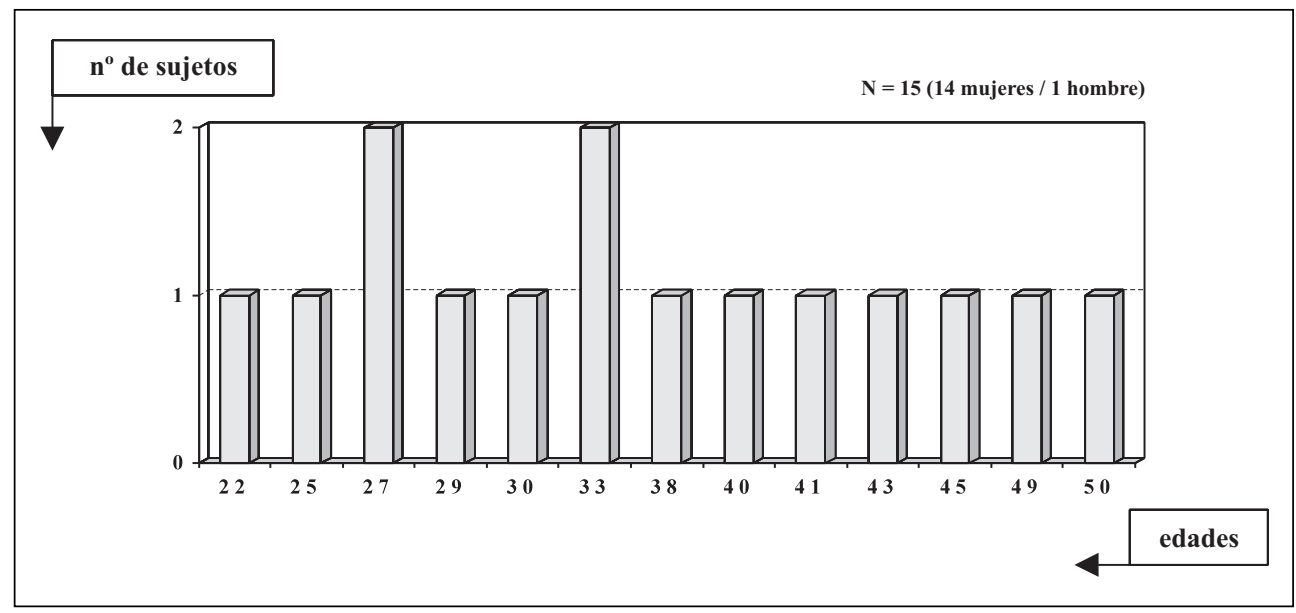

GRÁFICO 1.

Edad de los desempleados no universitarios orientados en el primer semestre del año 2000.

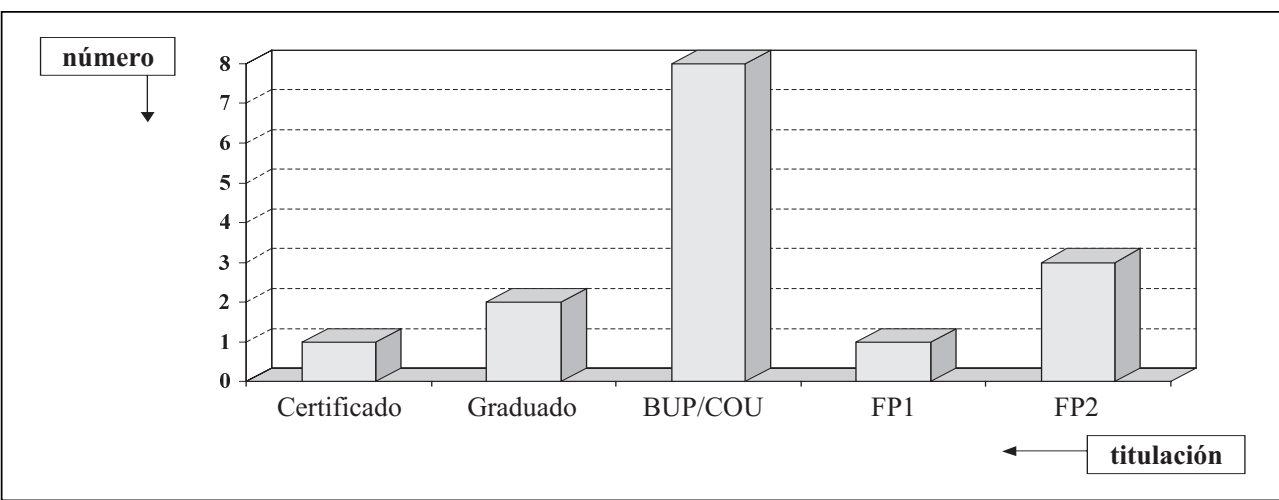

GRÁFICO 2.

Formación de los desempleados no universitarios orientados en el primer semestre del año 2000.

Por otra parte, hay otras que tienen más limitaciones a la hora de buscar activamente un empleo, expresan ciertas restricciones que las hacen inempleables, ya que buscan un trabajo para compatibilizar con otras facetas de su vida (generalmente dan más prioridad al ámbito familiar) y manifiestan que quieren alguna o varias de las siguientes opciones:

a) Un trabajo compatible con la recogida de los niños del colegio y el mantenimiento de las rutinas que le exige el hogar o la familia.

b) Lugar de realización cercano al hogar, que no les exija mucha dedicación ( $1 / 2$ jornada, tiempo parcial...). 
c) O dicen, que sólo necesitan algún dinero para contribuir, con un pequeño complemento, al gasto familiar.

Por otro lado, existe un subgrupo dentro de este grupo de no universitarios (que sobre todo han sido mujeres mayores de 40 años) que han requerido el Servicio de Análisis Vocacional, quizás porque, como podría comprobarse en sus narraciones, buscan en el trabajo la posibilidad de gozar, o lo que es lo mismo de desarrollar esa faceta expresiva.

Esta tesis, aplicable a algunas mujeres de cierta edad, nivel cultural medio-alto y sin necesidades económicas, que quieren trabajar y desarrollarse personalmente es avalada por Puchol (1994:114), quien nos comenta que:

"Los treinta y cinco o los cuarenta años (...) marcan un hito en nuestra existencia. Es la edad de la madurez, de la plenitud física, intelectual y emocional (...) aparte del dinero, buscan algo más, quieren responsabilidad, status y satisfacción en el trabajo realizado».

Curiosamente, hay actualmente, un interés por explotar estos años de la madurez, y, cada día, periódicos o revistas, sobre todo femeninas, muestran personajes «famosos» que han llegado a esta edad y hacen alarde de su vitalidad. En una revisión de las revistas semanales actuales encontramos algunas citas. Una de ellas se refiere a una entrevista realizada a la popular periodista Rosa María Mateo en la revista semanal Mujer de Hoy, y realizada por V. Drake (2000). Los comentarios de la entrevistada son ilustrativos, pues señala, en relación a su edad y el trabajo, lo siguiente:

«Para mí la vida empezó a ser relativamente maravillosa y no ha dejado de serlo a partir de los 40 (...) te das cuenta de que eres responsable de tus decisiones, eres una persona libre, y eso significa que puedes elegir, poder decir que no a lo que no te gusta».

Pero, no todas las mujeres de esta edad, porque no tenemos datos de hombres en este tramo de la escala, están convencidas de sus valores personales y profesionales; quizás, como consecuencia de haber vivido en una sociedad machista, en la que el suyo ha sido siempre un papel secundario, no encuentran esa fuerza interior que les ayude a salvar los obstáculos de esa búsqueda de empleo; lo que queda reforzado por el empleador que exige, cada vez más, formación, juventud y flexibilidad (con cierto grado de sometimiento). Esto explica que las personas que han utilizado el servicio de Motivación para el logro, han sido las de más edad, y en ningún caso las más jóvenes.

Sin embargo los demandantes más jóvenes han solicitado, fundamentalmente, los servicios de Información e Instrumentación, quizás porque tienen necesidad de trabajar de modo inmediato, y no ponen demasiadas limitaciones. Una vez más, se refuerza la tesis recogida por Blanch (Íb.: 119) donde se asocia juventud a búsqueda instrumental del trabajo (Gráfico 3).

\section{Orientados Universitarios}

El grupo de los 28 universitarios (26 mujeres y 2 hombres), que ha requerido la ayuda del servicio de Orientación de la Oficina de Empleo, tiene una edad comprendida entre los 22 y los 52 años, tal y como vemos en el gráfico 4.

Su formación es una muestra de las carreras que pueden realizarse en la Región de Murcia, ha sido, sobre todo, Psicología, Profesorado de EGB, Graduado Social y una larga lista 


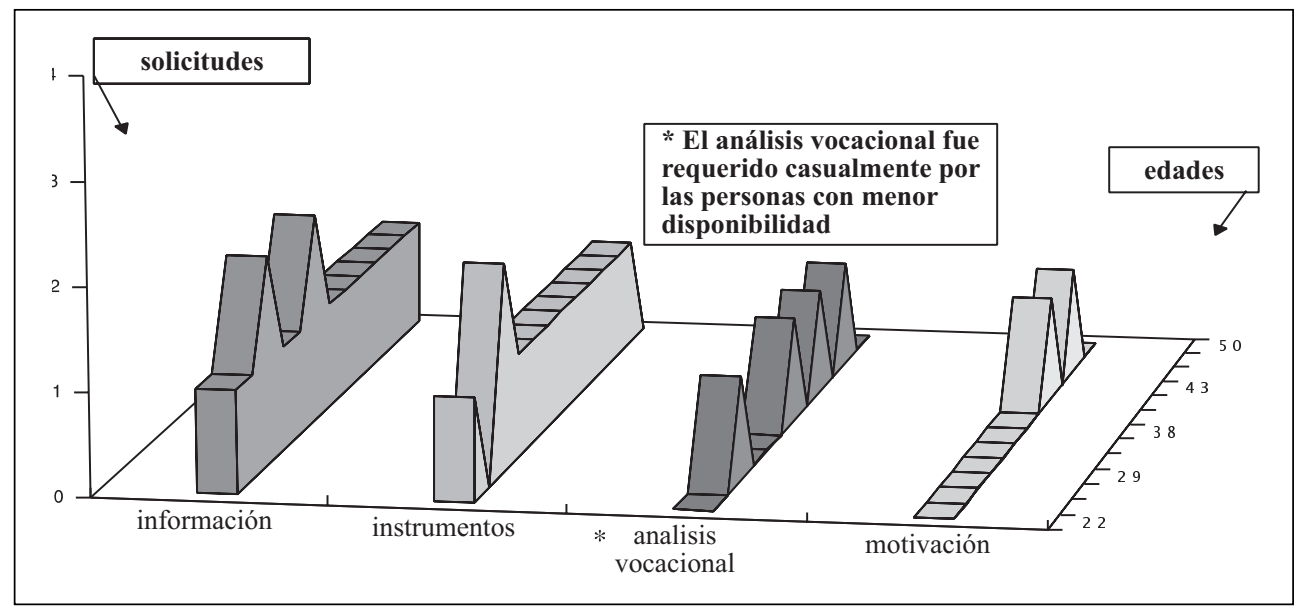

GRÁFICO 3.

Servicio de Orientación ofrecido según la edad.

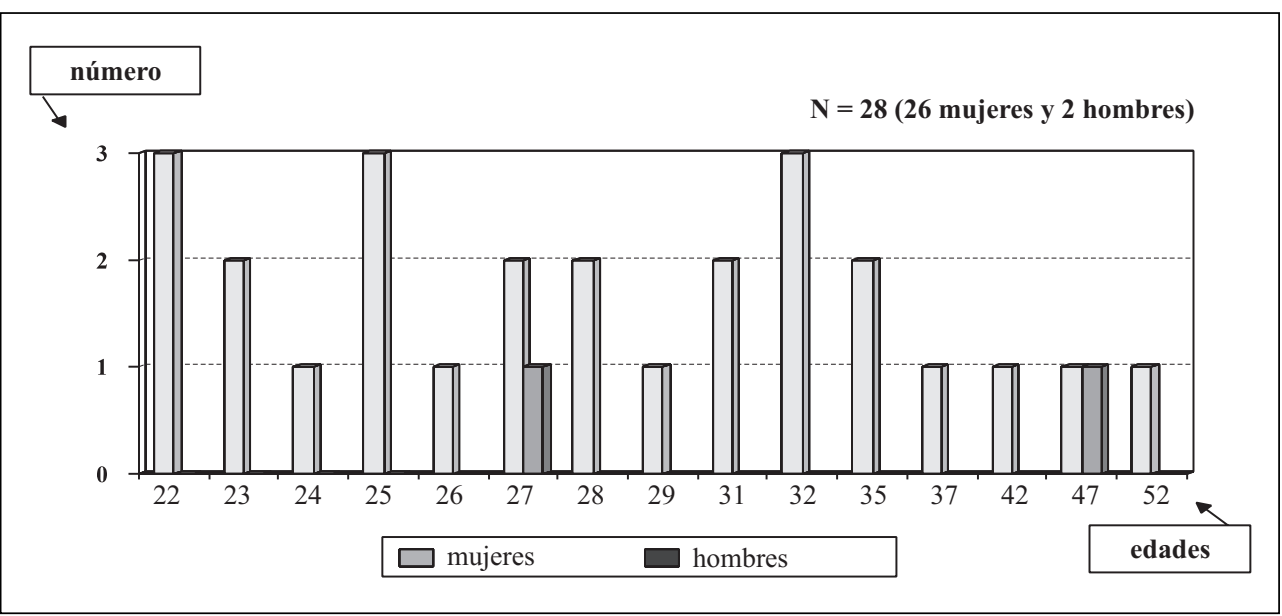

GRÁFICO 4.

Edad de los Orientados Universitarios en el ler. semestre del año 2000.

de otras especialidades -salvo Biología, Química y Enfermería-, relacionadas con las Ciencias Sociales o letras; que generalmente exigen al licenciado, después de que abandone la universidad, una «reconversión», a través de masters o cursos de especialización, que le permitan sobrevivir, entre la verdadera carrera de obstáculos que se le presenta cuando busca su primer empleo.

La experiencia profesional de éstos es más bien escasa, y generalmente viene marcada por la dedicación a trabajos poco cualificados, muy por debajo de su formación universita- 
ria. Parece ser que la necesidad de salir adelante, hace que en las primeras experiencias laborales, se busque en el trabajo, sobre todo, la función instrumental.

Pero veamos qué ocurre con las profesiones a las que optan. Es importante señalar que hay un grupo, de mujeres, que se incorpora a la rueda del mercado laboral después de una larga ausencia o, simplemente, después de que sus hijos hayan crecido, que opta curiosamente por trabajos muy por debajo de su formación, ya que consideran, según narran, que han perdido el tren, y que no pueden remontar la cuesta, incluso llegan a decir: «no se si me acordaría de eso que aprendí en la Universidad».

Así que deseosas de abandonar la rutina diaria necesitan un trabajo, sea como sea, y solicitan aquellos que creen que van a ofrecerles más oportunidades, como administrativo (que pueden «aprender» con algunos cursos Informática), conserje, telefonista, recepcionista, etc., convencidas de que no merece la pena empeñarse en mantener su status como universitarias.

La disponibilidad para incorporarse a un puesto de trabajo en estas personas, es muy alta. Tienen menos limitaciones que ningún otro colectivo, son - dicen-, conscientes de lo que quieren y pueden, van a por ello; no están apuntados al paro para ver que pasa después, sino que parece que quieren trabajar de inmediato; de ello da muestra el servicio de Orientación que han requerido, solicitan, sobre todo, Información, y sus preguntas suelen ser éstas: ¿cuáles son las Ofertas de trabajo con más posibilidades? ¿qué cursos de formación existen para este fin? o ¿cómo redacto mi curriculum vitae para que el empleador crea en mí? En el gráfico 5, se puede observar que han recibido fundamentalmente Información e Instrumentación, pero nunca Análisis Vocacional (quizás, porque ya tienen claro su objetivo profesional), y no necesitan, tampoco, Motivación para el logro.

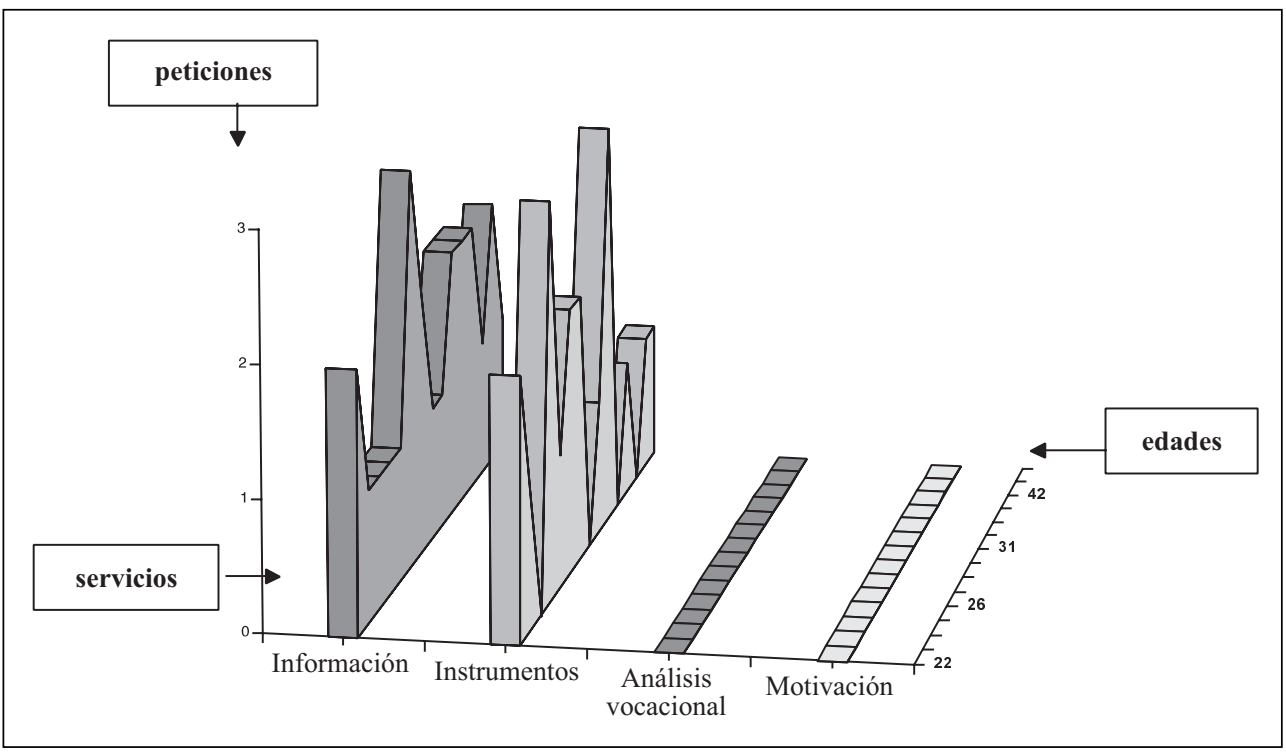

GRÁFICO 5.

Servicio de Orientación requerido por los Universitarios, ler. semestre del 2000. 


\section{Los Orientados preceptores de la Renta Activa para la Inserción (RAI)}

Este grupo es el único que no ha acudido al servicio de Orientación de forma voluntaria, siguiendo las instrucciones del Real Decreto 236/2000, de 18 de febrero, ya que al serles reconocido, durante 10 meses, su derecho a percibir una Renta (el 75\% del Salario Mínimo Interprofesional), están obligados a acudir al Servicio de Orientación.

Los datos que presentamos a continuación corresponden a las 65 personas, que han sido incorporados al Plan de Inserción Laboral (PIL) por tener reconocido su derecho a la RAI, en su primera convocatoria (ler. Semestre del año 2000). Su edad está comprendida entre los 45 y los 63 años, aunque un $80 \%$ de ellos se sitúa en el tramo de 45 a 52 .

El nivel académico de este grupo, aunque oscila entre Analfabetos y Licenciados, tiene su mayor porcentaje de Certificados de Escolaridad (la mayoría) y Graduados Escolares, ya que en ambas situaciones está casi el 90\% del grupo.

La experiencia profesional de este grupo es muy diversa, y va desde ocupaciones poco cualificadas como peones (una gran mayoría de los hombres y de las mujeres), ordenanzas, celadores, limpiadoras, así como oficios más especializados: auxiliar administrativo, electricista, cerrajero, conductor, pintor, etc.

Una curiosidad de este grupo es que la demanda de trabajo que solicitan, no suele ser continuidad a su experiencia anterior, ya que, generalmente, optan por puestos de menor especialización, poco riesgo, o generados por la administración a través de Convenios con el INEM, en los que éste -según el R.D. 236/2000-, es un grupo que debe seleccionarse con preferencia absoluta sobre cualquier otro.

Pero, también encontramos un pequeño número de demandantes, que no aceptan la RAI como medio de subsistencia, y solicitan un «lo que sea», incluso aunque cobraran lo mismo, con el fin de salir del paro, ya que consideran que sin trabajo la vida no tiene sentido para ellos.

Esta circunstancia no se da en el grupo de mujeres porque, quizás, en esa generación a la que pertenecen no está tan asumida, como en algunos hombres, la actividad laboral como rol vital, que ensalza el hecho de trabajar como un distintivo de moral y nobleza (Blanch, Íb.: 121).

Por otro lado, hay un gran número de perceptores RAI, tanto hombres como mujeres, que muestran baja disponibilidad para trabajar de forma inmediata. Unas veces se quejan, al ser llamados a trabajar, y dicen: "para una vez que tengo un Renta que me permite descansar un росо...», otras, aluden diversas limitaciones o minusvalías.

En diversos estudios analizados por Blanch (Íb.:198) se comprueba que hay una definición para aquellas personas que optan por «vivir (en la medida de lo posible) a costa de las prestaciones económicas y sociales del estado benefactor [...]» y que se señalan como "parásitos del bienestar»; mientras que nosotros, observando que se trata de gente humilde, consideramos que son personas que están hartas de trabajar de forma sumergida y penosa, por lo tanto, sin futuro, ni pensión de jubilación, ni fuerza en el cuerpo para seguir trabajando.

Otro dato peculiar de este colectivo es que sólo ha utilizado del servicio de Orientación, la Información, en particular, la referente a los cursos de Formación Ocupacional (F.O.), aunque, también es cierto que, una gran mayoría se resiste a iniciar un nuevo periplo forma- 
La Orientación Laboral de desempleados inscritos...

tivo en su vida. Por ello, sólo un $13 \%$ del total ha requerido esta información sobre cursos de diversa índole, y sólo uno de ellos ha solicitado información sobre las ayudas para crear su propia empresa.

\section{Conclusiones}

El desempleo y las personas que están en él nos muestran una gran diversidad de reacciones individuales, expectativas e intereses de cara a la búsqueda activa de empleo.

Esta idea es fundamental a la hora de realizar los programas de Orientación Laboral. Para una correcta elaboración hay que analizar las características del colectivo a tratar, sus intereses, posibilidades en el mercado laboral, expectativas, formación, edad, género; así se podrán organizar actividades que permitan reflexionar sobre posibles cambios, valorando sus efectos y alternativas.

Este estudio sobre la Orientación Laboral y los demandantes de la Oficina de Empleo de Murcia, nos permite concluir que, existe una amplia gama de perfiles de desempleados, que necesitan distintas ayudas. Combinando factores como edad, formación, experiencia profesional o género, observamos que:

a) Hay buscadores de empleo, que demandan información sobre salidas en el mercado laboral o cursos de especialización acordes a sus expectativas, además de modelos para la elaboración de currículos, o estrategias para participar, de forma inmediata, en entrevistas de selección. Entre ellos se encuentran universitarios o jóvenes con edades comprendidas entre los 18 y 35 años.

b) A otros grupos, formados por desempleados difíciles de emplear, debido a su edad, escasez de formación o falta de experiencia laboral y, consecuentemente, bajas expectativas de logro, les urge, como más prioritario, la participación en programas con actividades que le permitan, reconociendo sus limitaciones, mejorar su autoestima, y optimizar todos sus recursos personales y profesionales. Aquí se sitúan, sobre todo, mujeres con edades superiores a los 40 años o que se incorporan al mundo laboral después de la crianza de sus hijos y con una escasa formación profesional.

c) Para los desempleados, no buscadores, habría que disponer de información sobre subsidios, cursos, normativa legal que les acoge, etc., ya que no buscan en el servicio de Orientación ayuda para la búsqueda de empleo, sino para un mejor acomodo en el desempleo. En este grupo están las mujeres que compatibilizan trabajo y familia -a la que suelen dar preferencia-, y determinado grupo de personas mayores de 45 años aquejados de multitud de achaques por enfermedades o dolencias de diversa índole.

d) Por último, para las personas, que se incorporan al mercado laboral, después de un largo periodo de retiro, o despedidos de una empresa en la que han desempeñado una única tarea, generalmente obsoleta o mujeres que no necesitan el salario únicamente sino almo más, es necesario contar con instrumentos de análisis vocacional, que les permita analizar sus intereses, para que, conociendo las nuevas oportunidades del mercado laboral, clarifiquen sus objetivos e inicien nuevos rumbos. Aquí nos encontramos, sobre todo, a demandantes cercanos a los 40 años. 
Tomada así la Orientación laboral creemos que exige para llevarla a cabo profesionales que dispongan de recursos para adecuar los instrumentos o programas de orientación a su alcance a las características de cada colectivo. Además de conocer sus peculiaridades deben estar al tanto de las dificultades de insertarse en el mercado laboral de algunos de ellos (debido a edad, género, formación o experiencia), así como las ayudas existentes para la creación de empleo (subvenciones a la contratación, ayudas para el fomento de empleo autónomo o asociado), o las nuevas formas de trabajo (vía internet, franquicias, servicios novedosos). Esto les permitirá ofrecer la mayor variedad posible de opciones a sus clientes.

El análisis teórico y documental que acompaña al trabajo realizado está en consonancia con los datos empíricos encontrados, no obstante, queda mucho por hacer, ya que la Orientación Laboral en España está dando sus primeros pasos y necesita seguir analizando cada una de las variables que presenta su clientela.

La proliferación de programas -generalmente subvenciones del Fondo Social Europeo-, está ofreciendo una Orientación más o menos estandarizada que habrá que acomodar a cada grupo.

Habrá que seguir analizando las circunstancias y necesidades de cada uno de los grupos presentados, para ofrecer un servicio que responda a sus requerimientos. Aunque la gama de servicios y programas está empezando a diversificarse al amparo de diversas iniciativas -sobre todo dirigidas a grupos desfavorecidos: mujeres, inmigrantes, minusválidos, mayores de 45 años o jóvenes-, que parten del análisis del colectivo a ayudar, estudiando qué tiene y qué le falta para ofrecerle, como resultado, un instrumento de apoyo a su medida.

Paralelo a estos cambios ha aparecido la figura del orientador-tutor, cuyo cometido es adecuar el servicio o programa a las expectativas no sólo de cada grupo, sino incluso de cada cliente. Esperemos que este cambio hacia la individualización dé resultados positivos a corto plazo y, con ello, se amplíe y complete el marco teórico y práctico de la orientación laboral.

Por otro lado, y para futuras investigaciones, viendo la complejidad del mundo laboral, hay quien afirma que el servicio de Orientación deberá estar, también, junto a los que aún mantienen su empleo, tratando de prepararles para un futuro, quizás incierto, ya que, con toda seguridad, tendrán que pasar por actividades bien distintas, para mantener su empleo. Esta idea la recoge Puchol (1994: 115), recordándonos la curva sigmoide de Handy (1992):

"La curva sigmoide es una curva en forma de S, que puede representar la historia de la vida de un individuo. Comenzamos lentamente, posteriormente mejoramos, crecemos hasta alcanzar un máximo, tras el cual declinamos [...]. Afortunadamente, hay vida después de la curva, y el secreto de conseguir un continuo crecimiento y auge consiste en empezar una nueva curva sigmoide antes de que la anterior decaiga».

Además, el fenómeno de la inmigración está poniendo en evidencia tal vacío que, como consecuencia se han puesto en marcha, de modo espontáneo, una serie de cursos y masters que pretenden ayudar al orientador a conocer las nuevas premisas de un colectivo, hasta ahora desconocido. 


\section{Referencias bibliográficas}

Blanch, J. M. (1990). Del viejo al nuevo paro. Barcelona, PPU-INEM.

Dake, V. (2000). Maduras y encantadas. Mujer de Hoy. 70, pp. 4-6.

Goodale, J. G. (1982). La entrevista. Madrid, Ediciones Pirámide, 1988.

Puchol, L. (1994). Reorientación de carreras profesionales. Madrid, ESIC-EDITORIAL.

Real Decreto 236/2000 de 18 de febrero, B.O.E. de 10 de marzo de 2000.

Villamarzo, P. F. (1994), en M. Garrido Fernández. El trabajo desde la perspectiva psicoanalítica. Salamanca, Amarú ediciones.

Watt, G. (1997). El papel de la Orientación de Adultos y el Asesoramiento sobre Empleo ante la evolución del mercado de trabajo. Fundación Europea para la Mejora de las Comunidades Europeas. Luxemburgo.

Fecha de recepción: 17-VII-00

Fecha de revisión: 8-III-01

Fecha de aceptación: 5-II-02 